\title{
Effects of Different Times of Glutaraldehyde 2\% on Bacillus subtilis Spores (In Vitro)
}

\author{
Eshagh Lasemi ${ }^{1}$, Mohammad Hossain Kalantar Motamedi ${ }^{1}, 2$, Fina Navi ${ }^{1}$, Maryam Rezae ${ }^{3}$, Niousha \\ Homay Nikfar ${ }^{4}$, Zahra Danial ${ }^{2,5}$, Roojan Azizpour ${ }^{1}$
}

${ }^{1}$ Craniomaxillofacial Research Center, Tehran Dental Branch, Islamic Azad University, Tehran, Iran

${ }^{2}$ Trauma Research Center, Baqiyatallah University of Medical Sciences, Tehran, Iran

${ }^{3}$ Department of Microbiology, Dental Branch, Islamic Azad University, Tehran, Iran

${ }^{4}$ Private Practice

${ }^{5}$ Faculty of Psychology \& Educational Sciences, Allameh Tabataba'i University, Tehran, Iran

*Corresponding Author: Mohammad Hosein Kalantar Motamedi, M.D., Professor, Trauma Research Center, Baqiyatallah University of Medical Sciences, Tehran, Iran. Tel: +98-21-22616946, Email: motamedical@yahoo.com

Received February 20, 2017; Accepted October 29, 2017; Online Published November 29, 2017

\begin{abstract}
Background: Due to the importance of disinfectant and sterilization of dental instruments, in total, $14 \%-28 \%$ of dentists, $13 \%$ of assistants, and $17 \%$ of healthcare workers (HCWs) have been subjected to the hepatitis B virus (HBV), and more than 200 healthcare providers (HCPs) pass away annually in the United States from HBV infection catched from their work place. Objective: This study examined the effects of glutaraldehyde $2 \%$ on Bacillus subtilis spores in the Surgery and Microbiology Department of the Dental Branch of Islamic Azad University.

Methods: This experimental research evaluated a total of 58 samples, one called first evidence (pure glutaraldehyde not exposed to spore suspension), one called second evidence (spore suspension not exposed to glutaraldehyde), and 40 samples including a suspension with a normal turbidity of $1 \times 108 \mathrm{CFU} / \mathrm{mL}$ according to $0.5 \mathrm{McFarland}$ with exposure to glutaraldehyde $\%$. Experiments were done in time intervals of 10, 15, 20, 25, 30, 40, and 60 minutes with 8 repeats. In all times, a $B$. subtilis spore suspension was used as evidence and also as a case.

Results: This research was done on 58 samples. The results showed that in the 10th minute there were 102 colonies, $18.6 \pm 3.4$ in the 15th minute, $6.2 \pm 1.4$ in the 20th minute, $2.1 \pm 0.8$ in the 25 th minute, and no colonies after 30 minutes. In an overall observation, it was seen that there were more colonies in the first 10 minutes, and from 15 to 20 minutes, this amount significantly decreased; after 30 minutes in each 8 repeats, the growth of colonies had stopped completely, while in the evidence samples, B. subtilis spores grew.

Conclusion: It seems that the density of $2 \%$ glutaraldehyde in 30 minutes time was enough to destroy the spores of $B$. subtilis. Keywords: Glutaraldehyde, Bacillus subtilis Spores, Disinfection
\end{abstract}

\section{Background}

Healthcare providers (HCP) are in daily tangency with microorganisms in blood, saliva, laboratory specimens, and specimens which may possibly transfer infectious diseases. Persistent contact with microorganisms has extremely increased the morbidity rate of infectious diseases among HCP contrasted to that of society. ${ }^{1}$ It is assessed that $14 \%-28 \%$ of dentists, $13 \%$ of assistants, and $17 \%$ of healthcare workers (HCWs) have been subjected to the hepatitis B virus (HBV), ${ }^{2}$ and more than $200 \mathrm{HCP}$ in the United States die annually from $\mathrm{HBV}$ infection contracted from their work environment. ${ }^{3}$ Blood and saliva may port viruses, bacteria, and other pathogens that may reason diseases such as flu, HIV, pneumonia, tuberculosis (TB), HBV, and HIV. This peak the importance of infection rein at the workplace. ${ }^{1,4}$ The use of manners to delete or reduction the number of contaminants on instruments, pecimens, and tabletops is of higher importance. It is magistral to use bacterial solutions to delete or reduce the number of microorganisms on tools before autoclaving to exclude cross-infection. ${ }^{1}$ Reusable tools that are hand washed before processing can be hazardous. ${ }^{5}$

The results showed that the use of the disinfectant substance glutaraldehyde $2 \%$ for disinfecting the equipment of a urology operating room with respect to conditions and factors affecting disinfection, such as concentration, reaction time, and the correct disinfection procedures, completely removed Staphylococcus aureus, Citrobacter, Pseudomonas aeruginosa, Escherichia coli, and Klebsiella. Glutaraldehyde 2\% was effective on the studied pathogenic

Copyright $(2017$ The Author(s). This is an open-access article distributed under the terms of the Creative Commons Attribution License (http:// creativecommons.org/licenses/by/4.0), which permits unrestricted use, distribution, and reproduction in any medium, provided the original work is properly cited. 
microorganisms. ${ }^{6}$ Tools should be decontaminated and then sterilized to remove microbes and spores. ${ }^{2,6-8}$ Omidkhoda et al and Camilla et al evaluated the effects of different disinfection methods on orthodontic pliers. They reported that glutaraldehyde was an acceptable disinfectant agent., ${ }^{9,10}$ Simoes et al studied the effect of glutaraldehyde on the control of mono- and dual-species biofilms of Bacillus cereus and pseudomonas in 2011.11 Another study done in 2008 by Retta and Saripanti showed the structure of a mathematic model for anticipating the activity of glutaraldehyde in destroying spores. In this model, the effect of glutaraldehyde was calculated exactly by changing factors such as the density of glutaraldehyde, the temperature, and the influence time of the substance. ${ }^{12}$

\section{Objective}

Tools should be antisepted and then sterilized to eliminate microbes and spores. A literature search revealed no researches existing about disinfection of different instruments by glutaraldehyde $2 \%$; thus, this study evaluated the effects of this solution on Bacillus subtilis spores to determine whether glutaraldehyde $2 \%$ can be effective in disinfecting dental settings. Experiments were conducted in 2016 at the OMFS and Microbiology Department of the Dental Branch of Islamic Azad University.

\section{Methods}

A glutaraldehyde $2 \%$ solution was combined in this experimental study. B. subtilis spores ATCC6633 KD were purchased from the laboratory research center. A spore suspension with normal opacity $1 \times 10^{8} \mathrm{CFU} /$ $\mathrm{mL}$ was prepared using frustrated physiological serum in accordance with McFarland 0.5 standard. ${ }^{13}$ Then a sterilized certain tube was used for preparing the solution (controls). In other tube $2 \mathrm{~mL}$ of newly opened flacon of glutaraldehyde $2 \%$ was combined to the test tube using a spay pipette and the tube was encased; then $1 \mathrm{~mL}$ of the spore solution with the same standard opacity was added to the test tube (test group) containing glutaraldehyde $2 \%$. Because the producing factory claimed that glutaraldehyde $2 \%$ kills B. subtilis spores in 60 minutes, experiments were performed on nutrient agar at time intervals of $10,15,20$, $25,30,40$, and 60 minutes. ${ }^{14}$

For sampling, a standard ring was Catch fire for 10 seconds and then the specimens were prepared after the loop chilled. A sample from the liquid was taken at each of the aforesaid time - points and the conveyed to nutrient agar (NA) immediately (without contact with glutaraldehyde for controls). Then the cultures were placed in an incubator for 24 hours at $37^{\circ} \mathrm{C}$. Along with any series, a NA plate was placed inside the incubator and when no growth was looked, agar sterility was assured.

Experiments were repeated 8 times, until the growth consequence became negative. To ensure the absence of contamination, the standard loop was first placed in the flame for 10 minutes. Then, after the loop cooled, sampling was done. Furthermore, the suspension was cultured without adding glutaraldehyde $2 \%$, and then it was transferred to NA and placed in an incubator for 24 hours at $37^{\circ} \mathrm{C}$. Accompanying every set of samples into the incubator was a solid culture media. A lack of growth in this media confirmed sterilization.

The variables in this study included a plate of $B$. subtilis spores, glutaraldehyde $2 \%$, the time of substance (proximity time), growth or lack of growth after contact with the disinfectant, and environment. The data about growth or lack of growth of $B$. subtilis was surveyed using the Kruskal-Wallis and Friedman tests.

Numeric and qualitative variables were reported as mean $( \pm \mathrm{SD})$ and count $(\%)$, respectively. Kruskal-Wallis and Friedman tests were used for statistical analyses, and $P=0.05$ was considered statistically significant. SPSS version 17 was also used for statistical analyses.

\section{Results}

The results indicated that, in all 8 repetitions, at the time intervals of $10,15,20$, and 25 minutes, $10^{2}, 18.6 \pm 3.4,6.2$ \pm 1.4 , and $2.1 \pm 0.8$ colonies grew, respectively; after 30 minutes, no growth was observed. Overall, colonies were numerous up to 10 minutes, and from 15-25 minutes the number of colonies was severely reduced. At 30 minutes, there was no more growth. The procedure for each time interval was repeated 8 times and continued as long as the culture result was positive. The glutaraldehyde used in all procedures came from a single bottle that was opened on the first day. For sampling, a standard ring was Catch fire for 10 seconds and then the specimens were prepared after the loop chilled. A sample from the liquid was taken at each of the aforesaid time - points and the conveyed to NA immediately (without contact with glutaraldehyde for controls) (Figure 1). Then the cultures were placed in an incubator for 24 hours at $37^{\circ} \mathrm{C}$. Along with any series, a NA plate was placed inside the incubator and when no growth was looked, agar sterility was confirmed.

\section{Discussion}

Eliminating or reducing the number of microorganisms from reusable tools before autoclaving is significant in preventing cross-infection; in many offices reusable tools are still scraped before procedures. ${ }^{5}$ This may be perilous;

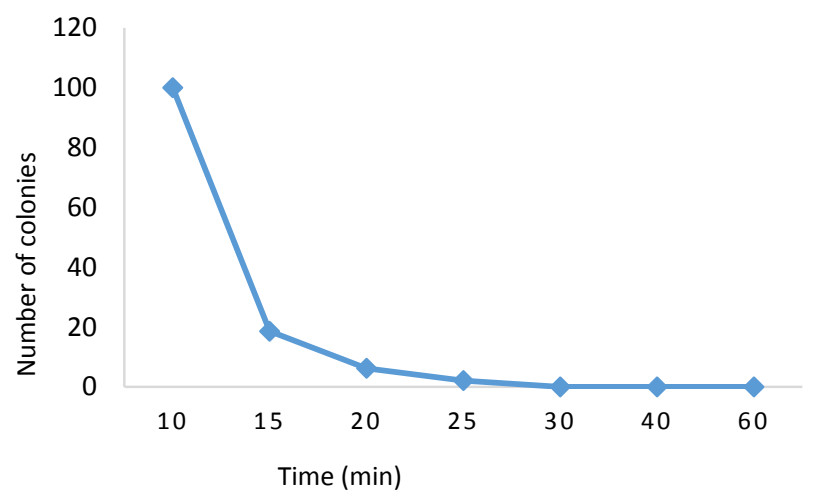

Figure 1. Colony Growth by Time Point $(n=8)$. 
reusable tools should be fumigated and then sterilized via autoclave, gamma beam or ethylene oxide to remove the microbes and insistent spores. The spore is the most insistent from of the microbe. Bacterial spores are among the most insistent of all living cells to biocides. ${ }^{15}$ In a 2010 study performed to find a solution of the high level disinfectant aldelo to eliminate mycobacterial and bacterial spores, Miner et al investigated glutaraldehyde and orthophthalaldehyde solutions with different concentrations of alcohol, sodium, and potassium, Cailliet compounds and detergents with an alkaline $\mathrm{pH}$ and evaluated them on mycobacteria and bacterial spores in order to eliminate bacteria in an appropriate time and a temperature between $20-25^{\circ} \mathrm{C}$. A concentration of less than $20 \%$ isopropanol, less than $8 \%$ potassium, and potassium acetate in composition with a lower concentration of glutaraldehyde $3.5 \%$ with an alkaline $\mathrm{pH}$ eliminated $6 \log _{10}$ mycobacteria in 10 minutes and at a temperature of $20^{\circ} \mathrm{C}$. Similar solutions eliminated $6 \log _{10}$ B. subtilis in 30 minutes at a temperature of $25^{\circ} \mathrm{C}$ and in 60 minutes at $20^{\circ} \mathrm{C}$. Ortho-phthalaldehyde spore paper properties did not increase in combination with isopropanol and potassium acetate. The researchers also stated that a high level disinfectant with the formulation of glutaraldehyde $5.3 \%$ in combination with isopropanol $20 \%$ and potassium acetate $8 \%$ eliminated mycobacteria in 10 minutes at a temperature of $20^{\circ} \mathrm{C}$ and $6 \log _{10}$ Bacillus subtilis spores in 60 minutes at $20^{\circ} \mathrm{C}$. The results of this study were similar to those of the current study with one difference; in Miner et al, a temperature between $20-25^{\circ} \mathrm{C}$ was considered, but the current study was done at room temperature. ${ }^{16}$

da Silva et al evaluated the effects of 6 different disinfectants on the elimination of five species of bacteria and their effects on a resin acrylic surface texture. Performed in 2007, their study aimed to highlight the effects of disinfectant (sodium hypochlorite $1 \%$, chlorhexidine digluconate $2 \%$, chlorhexidine $2 \%$, vinegar $100 \%$, and a denture cleaning pill with a perborate sodium base and perborate sodium 3.8\%) in disinfecting acrylic resin samples $(\mathrm{n}=10)$ contaminated with Candida albicans, Streptococcus mutans, S. aureus, E. coli, and B. subtilis; the remaining colonies were measured in CFU in a laboratory. In another study, acrylic resin was affected with disinfectant in order to evaluate its probable tag which may lead to microbe accumulation on its surface. A total of 3050 species of acrylic resin were contaminated in a $1 \times 10^{6}$ cells/ $\mathrm{mL}$ solution for 15 minutes. The control group was not affected with any disinfectant. The final microorganism count was done with the plating method to evaluate the reduction in microbes. da Silva et al studied 60 species to evaluate the effects of disinfectant on sample surfaces. Their conclusions showed that sodium hypochlorite $1 \%$, glutaraldehyde $2 \%$, and chlorhexidine digluconate $2 \%$ had the maximum effect on microorganisms followed by vinegar $100 \%$, sodium perborate $3.8 \%$, and pills with a sodium perborate base (used for denture cleaning). ${ }^{14}$ Their results were similar to those of the current study.
Research Highlights

\section{What Is Already Known?}

It is known that $14 \%-28 \%$ of dentists, $13 \%$ of assistants, and $17 \%$ of HCW have been encountered to HBV.

\section{What This Study Adds?}

This study shows that the density of $2 \%$ glutaraldehyde in 30 minutes was enough to destroy Bacillus subtilis spores.

They evaluated glutaraldehyde $2 \%$, which was similar to the base in the current study, and the type of spore was also similar. In both studies, immersion was performed, but da Silva et al experimented on acrylic resin, while the aim of the current study was dental instruments.

Simoes et al studied the effects of glutaraldehyde on the control of mono- and dual-species biofilms of B. cereus and Pseudomonas in 2011. Their results showed that in the dual-species biofilms of metabolic activity, the frequency of cells and the amount of matrix proteins were more than in both the single sampling and the inactivation of the single biofilm. Both kinds were the same; however, the dualspecies biofilm was the stronger kind. Glutaraldehyde 200 $\mathrm{mg}(-1)$ destroyed a small amount of the biofilm $(<10 \%)$. Finally, this study concluded that glutaraldehyde had a notable effect on Pseudomonas fluorescens and B. cereus. ${ }^{11}$

In 2014, Navi and Dejahang evaluated the effects of time and prolongation of Nanosil 6\% on B. subtilis spores in the Dental Branch of Tehran Azad University of Dentistry. In this in vitro study, to determine the time of the Nanosil effect, 33 samples (3 samples of pure Nanosil without spore exposure, 3 samples of spore suspension without Nanosil exposure, and 27 samples containing spore suspension with a normal turbidity of $1 \times 10^{8} \mathrm{CFU} / \mathrm{mL}$ according to the McFarland 0.5 standard with Nanosil) were investigated at 8 times (30, 60, 120, 180, 240, 300, 360, and 420 minutes). The study also investigated 114 samples of pure Nanosil without spore suspension exposure, 30 samples of spore suspension without Nanosil exposure, and 54 samples of spore suspension with Nanosil exposure in 10 days of follow-up. One of the reasons that glutaraldehyde is effective is the high level of disinfectant and the short time needed for it to take effect. ${ }^{17}$

\section{Conclusion}

It seems that the density of $2 \%$ glutaraldehyde in 30 minutes time was enough to destroy $B$. subtilis spores. It is suggested that further research be done on higher level densities in order to destroy spores more strongly and quickly. It is also suggested that research be done on the shelf life of the solution and the reduction in its effect over time.

\section{Authors' Contributions}

Study design: LD, MHKM; Data collecting: FN; Data analysis: RA, MR; Manuscript preparation: MHKM, FN, ZD. 


\section{Conflict of Interest Disclosures}

The authors declared no potential conflicts of interest with respect to the research, authorship, and/or publication of this article.

\section{Ethical Approval}

\section{Not applicable.}

\section{References}

1. Farahani M, Sanaei AS. Infection control at dental office. Tehran: Baraye Farad; 2008:13-45. [Persian].

2. Heymann HO, Swift EJ Jr, Ritter AV. Sturdevant's Art and Science of Operative Dentistry. 6th ed. London: Mosby; 2012: 376-81.

3. Etesam F. Principle of Biology. Tehran: Islamic Azad Universuty, Tehran Dental Branch; 1986:1-6. [Persian].

4. Adibfar P. Medical Microbiology. Tehran: Nor-e-Danesh Co; 2004:279-289. [Persian].

5. Sepkowitz KA. Occupationally acquired infections in health care workers. Part II. Ann Intern Med. 1996;125(11):917-928. doi:10.7326/0003-4819-125-11-199612010-00008.

6. Alamdari A, Behrouzi M, Hosseini SM, Sadeghi H, Azizi A, Ghafarian Shirazi HR. Comparison of two disinfectant solutions deconex 53 plus 2\% and glutaraldehyde (Cidex) $2 \%$ on reduction of medical equipment contamination in the operating room of urology ward. Ann Trop Med Public Health. 2017;10(4):910-913.

7. Coates D. Sporicidal activity of sodium dichloroisocyanurate, peroxygen and glutaraldehyde disinfectants against Bacillus subtilis. J Hosp Infect. 1996;32(4):283-294. doi:10.1016/ S0195-6701(96)90039-0.

8. Anderson TR, Slotkin TA. Maturation of the adrenal medulla--IV. Effects of morphine. Biochem Pharmacol. 1975;24(16):14691474. doi:10.1016/0006-2952(75)90020-9.
9. Omidkhoda M, Rashed R, Bagheri Z, Ghazvini K, Shafaee H. Comparison of three different sterilization and disinfection methods on orthodontic markers. J Orthod Sci. 2016;5(1):1417. doi:10.4103/2278-0203.176653.

10. de Almeida CMF, de Carvalho AS, Duarte DA. Evaluation of disinfection methods of orthodontic pliers. Dental Press J Orthod. 2012;17(4):105-109. doi:10.1590/S217694512012000400020 .

11. Simoes LC, Lemos M, Araujo P, Pereira AM, Simoes M. The effects of glutaraldehyde on the control of single and dual biofilms of Bacillus cereus and Pseudomonas fluorescens. Biofouling. 2011;27(3):337-346. doi:10.1080/08927014.201 1.575935

12. Retta SM, Sagripanti JL. Modeling the inactivation kinetics of bacillus spores by glutaraldehyde. Lett Appl Microbiol. 2008;46(5):568-574. doi:10.1111/j.1472-765X.2008.02358.x.

13. Miller C, Palenic C. Infection Control and Management of Hazardous Material for Dental Team. 2nd ed. St. Louis: Mosby; 1998:3-6.

14. da Silva FC, Kimpara ET, Mancini MN, Balducci I, Jorge AO, Koga-Ito CY. Effectiveness of six different disinfectants on removing five microbial species and effects on topographic characteristics of acrylic resin. J Prosthodont. 2008;17(8):627633. doi:10.1111/j.1532-849X.2008.00358.x.

15. Russell AD. Bacterial spores and chemical sporicidal agents. Clin Microbiol Rev. 1990;3(2):99-119. doi:10.1128/ CMR.3.2.99.

16. Miner N, Harris V, Cao TD, Ebron T, Lukomski N. Aldahol highlevel disinfectant. Am J Infect Control. 2010;38(3):205-211. doi:10.1016/j.ajic.2009.08.009.

17. Navi F, Dejahang S. Comparison of concentration of $2 \%$ and $6 \%$ Nanosil on Bacillus subtilis spores (in vitro) [dissertation]. Tehran: School of dentistry, Azad University of Tehran; 2014. [Persian]. 